\title{
Fabrication and Photocatalytic Properties of ZnSe Nanorod Films
}

\author{
Jiajia Yin, ${ }^{1}$ Chao Yang, ${ }^{1}$ Xianheng Yang, Shutong Wang, ${ }^{1}$ Hua Zhang, \\ Shouhuan Zhou, ${ }^{1,2}$ and Guoying Feng ${ }^{1}$ \\ ${ }^{1}$ Institute of Laser \& Micro/Nano Engineering, College of Electronics \& Information Engineering, Sichuan University, \\ Chengdu, Sichuan 610065, China \\ ${ }^{2}$ North China Research Institute of Electro-Optics, Beijing 100015, China \\ Correspondence should be addressed to Shouhuan Zhou; zhoush@scu.edu.cn and Guoying Feng; guoing_feng@scu.edu.cn
}

Received 25 February 2016; Accepted 6 June 2016

Academic Editor: Jeffrey Elam

Copyright (C) 2016 Jiajia Yin et al. This is an open access article distributed under the Creative Commons Attribution License, which permits unrestricted use, distribution, and reproduction in any medium, provided the original work is properly cited.

\begin{abstract}
ZnSe nanorod films grown on fused quartz glass substrates via a simple two-step synthesis protocol were demonstrated to be environmentally safe and effective recyclable photocatalysts. These films showed greatly enhanced photocatalytic activity compared to pulsed laser deposition ZnSe films in the degradation of methyl orange dye solutions. The well-crystalized ZnSe nanorods had a length of $15 \mu \mathrm{m}$ and a diameter of $200 \mathrm{~nm}$ and were densely grown on the substrate. The morphology, crystal structure, crystal phase, and photophysical properties of the ZnSe nanorod films were investigated using field-emission scanning electron microscopy (FESEM), UV-Vis spectroscopy, X-ray diffraction (XRD), transmission electron microscopy (TEM), and high resolution transmission electron microscopy (HRTEM)
\end{abstract}

\section{Introduction}

The interest in fabricating of one-dimensional (1D) ZnSe nanostructures such as nanorods [1], nanobelts [2], nanowires [3], and nanoneedles [4] has increased over the decades partly due to their potential uses as active components in the preparation of blue-ultraviolet photodetectors [2], light-emitting diodes [5], photo-voltaic solar cells [6], and more. In addition, possible quantum confinement effects and the large specific surface area of $1 \mathrm{D}$ ZnSe nanostructures have driven investigations of photocatalytic activity $[7,8]$. Unfortunately, nanostructured photocatalysts are prone to aggregation (decreasing the photoactive surface area) and are difficult to collect for reuse, so studies on the renewable photocatalytic properties of $1 \mathrm{D} \mathrm{ZnSe}$ nanostructure photocatalysts are rarely performed $[6,9,10]$. However, supporting the photocatalytic materials with a rigid substrate enables practical photocatalytic application $[11,12]$.

There are various methods available for the fabrication of 1D ZnSe nanostructures films, solvothermal method [13], template method [14], atomic layer deposition (ALD) [15], molecular beam epitaxy (MBE) [16], and metal-organic vapor phase epitaxy (MOVPE) $[5,17,18]$. However, these methods generally (a) require extremely conditions, (b) can easily become contaminated, and (c) are difficult to control.

In the presented work, we present a simple two-step synthesis protocol for the fabrication of a $\mathrm{ZnSe} \mathrm{nanorod} \mathrm{films.}$ The first step is a pulsed laser deposition (PLD) of a ZnSe thin film, and the second step is an ultrasonic-assisted thermal treatment to promote $\mathrm{ZnSe}$ nanorod growth. This method is very simple and effective and enables the production of impurity-free $\mathrm{ZnSe}$ nanorod films. Considering cost for mass production, we used fused quartz glass as the substrate in this study. Furthermore, we used the $\mathrm{ZnSe}$ nanorod films as a recyclable photocatalysts. As a comparison, the photocatalytic activities of the ZnSe nanorod film and the ZnSe film in response to ultraviolet excitation light were examined.

\section{Experiment}

2.1. Preparation of ZnSe Nanorod Films and ZnSe Film. ZnSe crystal (high purity, 99.99\%) was used as a source material for 


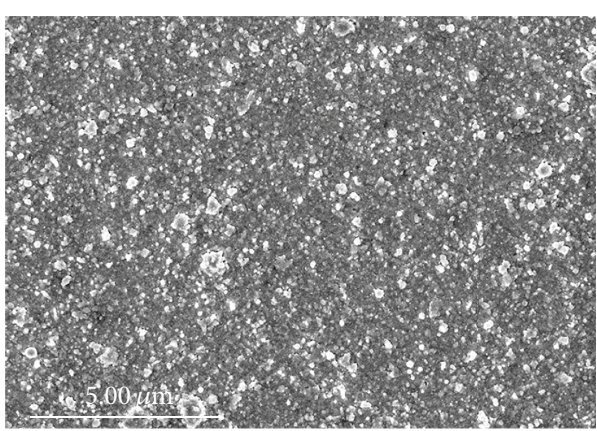

(a)

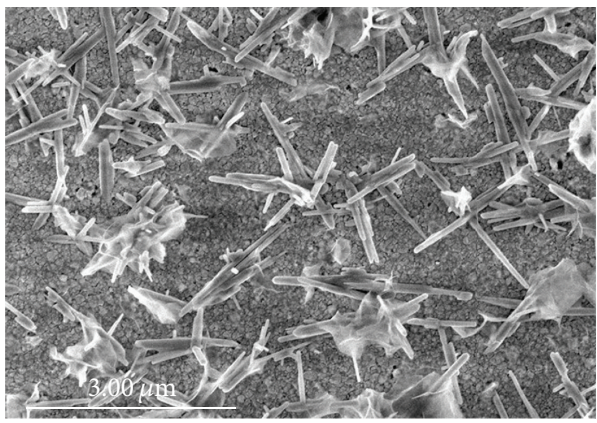

(c)

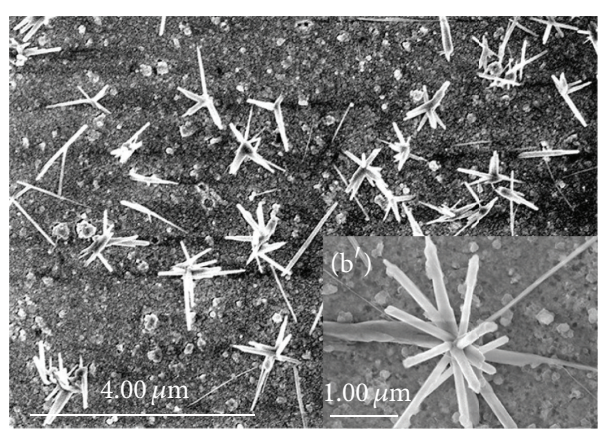

(b)

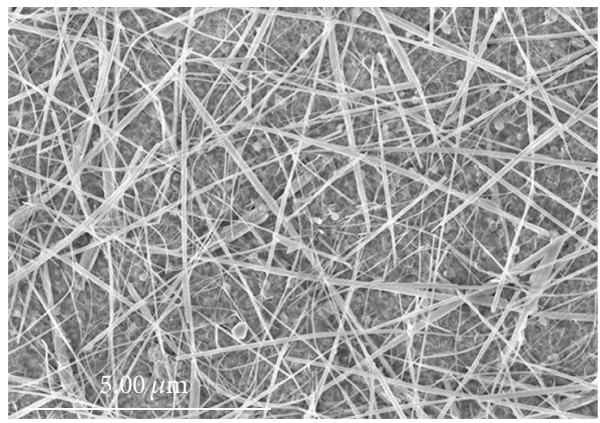

(d)

FIGURE 1: SEM images of (a) ZnSe film prepared using the pulsed laser deposition method. (b), (c), and (d) are the ZnSe film after ultrasonicassisted thermal treatment for $4 \mathrm{~h}, 8 \mathrm{~h}$, and $12 \mathrm{~h}$, respectively. The inset of (b) is an enlarged image detailing the nanorods growth.

the pulsed laser deposition of ZnSe films on fused quartz glass substrates. Before deposition of thin film, the fused quartz glass substrates were ultrasonically cleaned sequentially in acetone and alcohol and washed in running deionized (DI) water for $10 \mathrm{~min}$ and then dried in air. In order to guarantee film uniformity, the fused quartz glass substrates were clamped on a rotatable mounting plate. During deposition, the vacuum chamber was evacuated to a pressure below 1 $\times 10^{-7}$ Torr by a mechanical pump and a molecular. Then the fused quartz glass substrate was heated up to $450^{\circ} \mathrm{C}$. The target was ablated by a titanium doped sapphire regenerative amplified mode-locked femtosecond laser (Coherent Inc., with a center wavelength of $800 \mathrm{~nm}$, pulse width of $\sim 45 \mathrm{fs}$ and $1 \mathrm{kHz}$ repetition rate). The laser fluence focused on the target was $0.16 \mathrm{~J} / \mathrm{cm}^{2}$. The deposition time was $30 \mathrm{~min}$ and the distance between target and substrate was $8.0 \mathrm{~cm}$. At the first process, we get the ZnSe film. After that stage, one of the well prepared ZnSe films was put into a temperaturecontrolled water (deionized water) bath having a constant temperature of $60^{\circ} \mathrm{C}$ and executed with ultrasonic-processing continuously in the thermal treatment process. During the ZnSe nanorod films formation process, the films were taken out to be analyzed at various durations. After the two-step synthesis route, we get the $\mathrm{ZnSe}$ nanorod films.

2.2. Photocatalytic Experiments. The photocatalytic activity of $\mathrm{ZnSe}$ nanorod films and $\mathrm{ZnSe}$ film with identical mass $(1.47 \mathrm{mg})$ of $\mathrm{ZnSe}$ on fused quartz glass substrates was investigated using methyl orange (MO) dye $\left(5 \times 10^{-5} \mathrm{M}\right)$.
The samples were immersed into $5 \mathrm{~mL}$ of the MO dye solution and irradiated with ultraviolet light (UV) at room temperature. Using a power meter, the incident light intensity was measured to be $5 \mathrm{~mW} \mathrm{~cm}$. Also the photoluminescence intensity was used to evaluate the variation of the MO's concentration. It was determined by a Hitachi F-4500 fluorescence spectrophotometer.

2.3. Characterization of the ZnSe Nanorod Films and $\mathrm{ZnSe}$ Film. Field-emission scanning electron microscopy (FESEM) images were obtained with a field-emission microscope (Hitachi SU-8220, acceleration voltage $3 \mathrm{kV}$ ). The optical transmission of the film was recorded at room temperature in the wavelength range of $400-1100 \mathrm{~nm}$ using Hitachi F-4500 fluorescence spectrophotometer. X-ray powder diffraction (XRD) patterns were collected by a Japan Rigaku D/max-rB X-ray diffractometer with $\mathrm{Cu} \mathrm{K} \alpha$ radiation $(\lambda=0.154178 \mathrm{~nm})$, operated at $80 \mathrm{~mA}$ and $40 \mathrm{kV}$. Hitachi $\mathrm{H}-$ 800 transmission electron microscope was used to observe transmission electron microscopy (TEM) image; it was working at an acceleration voltage of $200 \mathrm{kV}$.

\section{Results and Discussion}

Figure 1 shows SEM images of the ZnSe films following ultrasonic-assisted thermal treatment for different time length. As shown in Figure 1(a), the original $\mathrm{ZnSe}$ film prepared using the PLD method had a relatively dense and 


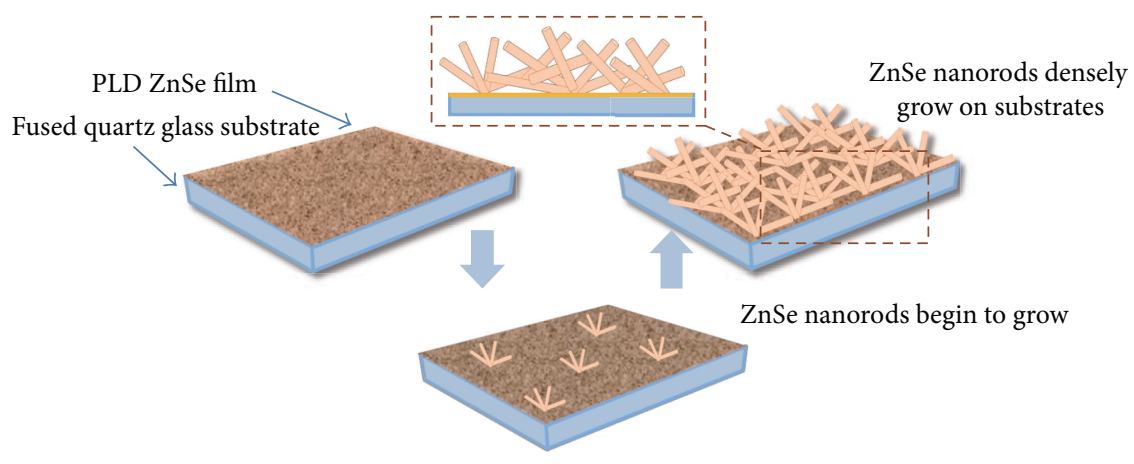

FIGURE 2: Schematic of the ZnSe nanorod film formation process.

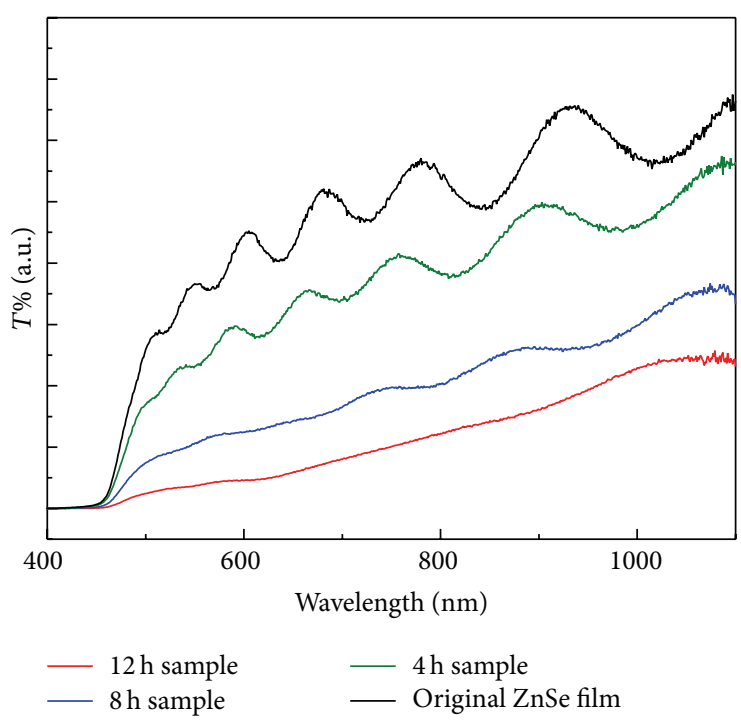

(a)
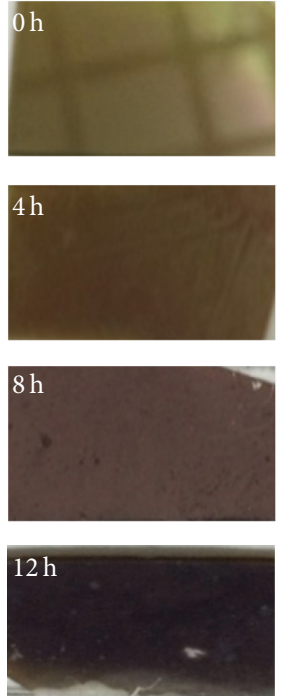

(b)

FIgURE 3: (a) Optical transmission of ZnSe film prepared using the PLD method and the ZnSe film after ultrasonic-assisted thermal treatment for $4 \mathrm{~h}, 8 \mathrm{~h}$, and $12 \mathrm{~h}$, respectively. (b) The photos behind are the corresponding ZnSe film treatment for $0 \mathrm{~h}, 4 \mathrm{~h}, 8 \mathrm{~h}$, and $12 \mathrm{~h}$.

smooth surface texture. However, there were large particles on the film surface indicative of the PLD technique [19]. A likely nucleation and growth mechanism is that as the ultrasonic-assisted thermal treatment time increased, the ZnSe nucleated. The prepared nanoparticles found their preferred deposition site because the liquid surface had a large accommodation coefficient. Rapid crystal growth took place when stable ZnSe nuclei were formed. Interaction forces between atoms in different nanoparticles induced translational and rotational movement, driving the nanoparticles into different locations and orientations for aggregation [3, 20]. As a result, nanorods began to grow on the $\mathrm{ZnSe}$ film as shown in Figure 1(b). The inset SEM image from Figure 1(b) shows that the nanorods clearly grew from a point, and Figure 1(c) shows that these rods lengthened as time passed. As shown in Figure 1(d), randomly oriented $\mathrm{ZnSe}$ nanorods densely covered the $\mathrm{ZnSe}$ film at the final stage. The network-like nanorod structure has a high surfaceto-volume ratio and interconnected open pores, which can remarkably enhance photodegradation. The average length and diameter of the nanorods were approximately $15 \mu \mathrm{m}$ and $200 \mathrm{~nm}$, respectively. A schematic of the ZnSe nanorod film formation process is shown in Figure 2.

The surface morphology transformation of the $\mathrm{ZnSe}$ film following the ultrasonic-assisted thermal treatment can be characterized through the change of optical transmission. Figure 3(a) shows the optical transmission of the ZnSe film as a function of wavelength. The four lines correspond to the ZnSe film following ultrasonic-assisted thermal treatment at time intervals $0 \mathrm{~h}, 4 \mathrm{~h}, 8 \mathrm{~h}$, and $12 \mathrm{~h}$, respectively. The thickness of the original PLD ZnSe film can be calculated by fitting the transmission data of spectrum (a) to the following equation [21]:

$$
\begin{aligned}
2 n_{i} d & =m \lambda_{i}, \\
2 n_{j} d & =(m+1) \lambda_{j}, \\
d & =\frac{\lambda_{i} \lambda_{j}}{2\left(n_{j} \lambda_{i}-n_{i} \lambda_{j}\right)},
\end{aligned}
$$




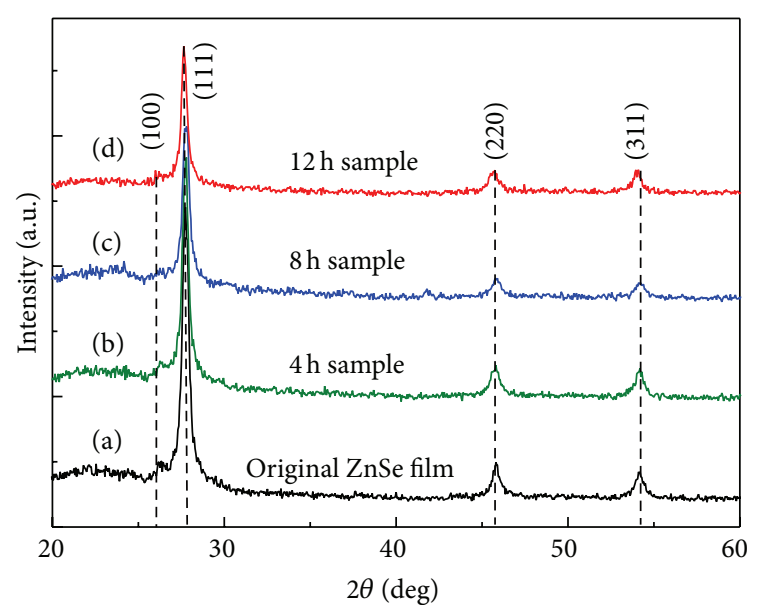

FIGURE 4: XRD patterns of (a) ZnSe film prepared using the PLD method. (b), (c), and (d) are the XRD patterns of the ZnSe film after ultrasonic-assisted thermal treatment for $4 \mathrm{~h}, 8 \mathrm{~h}$, and $12 \mathrm{~h}$, respectively.

where $n$ is the index of refractive, $d$ is the film thickness, $m$ is the interference order, and $\lambda$ is the wavelength. The indexes $i$ and $j$ were used to label the corresponding parameters (e.g., wavelength and refractive index) of neighbor peaks (maximum value). The results show that the thickness of the original PLD ZnSe film was approximately $1.21 \mu \mathrm{m}$. Comparing these four spectra, the interference peaks in (a) and (b) indicate that the PLD ZnSe film was relatively uniform at the initial stage. As ultrasonic-assisted thermal treatment time increased, the ZnSe film optical transmission decreased, and the interference peaks in the spectrum gradually disappeared. Also as shown in Figure 3(b), the films are becoming darker with increasing anneal time. That is because the $\mathrm{ZnSe}$ nanorods began to grow and the density of the nanorods increased. These changes resulted in the absorption coefficient of $\mathrm{ZnSe}$ nanorods film increased and then lead to the decrease of the optical transmission.

Figure 4 shows the structural characterization of the ZnSe film prepared using the PLD method and the samples following ultrasonic-assisted thermal treatment for $4 \mathrm{~h}, 8 \mathrm{~h}$ and $12 \mathrm{~h}$. Compare the four XRD patterns; there were three dominant diffraction peaks. The highest intensity reflection peak was at $2 \theta=27.71$ (111), and another two small intensity peaks were at $2 \theta=45.92(220)$ and 54.22 (311). Also, one small peak at $2 \theta=26.3(100)$ was observed. It is possible that some hexagonal phase (JCPDS 15-105) ZnSe is present in the four films along with the cubical phase (JCPDS 37-1463) ZnSe. This may be a result of the relatively low growth temperature used during the PLD ZnSe film fabrication process. Some researchers have reported that, at growth temperatures lower than $475^{\circ} \mathrm{C}$, ZnSe hexagonal crystallites will show cubic ZnSe films [22]. In the four XRD patterns, no other diffraction peaks were found, indicating that the ZnSe film and ZnSe nanorod film were free of crystalline impurities.
ZnSe nanorods were taken from the film and further investigated using TEM, as shown in Figure 5(a). Clearly, the $\mathrm{ZnSe}$ nanorod is relatively uniform. More information was derived from the HRTEM image and selected-area electrondiffraction (SAED) pattern and shown in Figure 5(b). The results demonstrate that the ZnSe nanorods on the film were well crystalized, with an internal atomic lattice spacing of $0.33 \mathrm{~nm}$ and $0.20 \mathrm{~nm}$ corresponding to the (111) and (220) planes of cubic ZnSe, respectively. Additionally, the growth direction of the nanorods was determined to be [220]. The SEM elemental mapping, shown in Figures 5(c)-5(f), further confirmed the formation of the $\mathrm{ZnSe}$ nanorods.

Organic dyes are widely used to color products in the textile, plating, and printing industries. Many dyes can form highly toxic complexes with heavy metal ions in wastewater and pollute water resources $[23,24]$. Photocatalytic degradation is considered one of the most efficient and economical methods for water purification.

The photocatalytic activity of the ZnSe nanorod film (following $12 \mathrm{~h}$ of ultrasonic-assisted thermal treatment) and the $\mathrm{ZnSe}$ film was examined through the degradation of methyl orange $(\mathrm{MO})$ dye in aqueous solution $\left(5 \times 10^{-5} \mathrm{M}\right)$ at various time durations. The $\mathrm{ZnSe}$ nanorod film and $\mathrm{ZnSe}$ film were immersed in the MO dye solution and irradiated with UV light under the same conditions. As shown in Figures 6(a) and 6(b), the photocatalytic degradation process was detected using Hitachi F-4500 fluorescence spectrophotometer. The characteristic absorption of the MO dye at $\lambda=462 \mathrm{~nm}$ was selected to monitor the photocatalytic degradation process. As illustrated in Figures 6(a) and 6(b), roughly 60\% of the MO dye decomposed after exposure to $3 \mathrm{~h}$ of UV light in the presence of the $\mathrm{ZnSe}$ nanorod film. Roughly $18 \%$ of the MO dye decomposed after exposure to $3 \mathrm{~h}$ under UV light in the presence of the $\mathrm{ZnSe}$ film. The variation of $\mathrm{MO}$ dye relative concentration with respect to irradiation time is shown in Figure 6(c). The control experiments indicated that the degradation of the MO dye was negligible without UV light irradiation or without photocatalysts. It was found that the degradation efficiency in the presence of the ZnSe film was low. On the contrary, the ZnSe nanorod film showed strong adsorptive capacities and comparable photocatalytic activities. This high photocatalytic activity of ZnSe nanorod film might be the result of the larger specific surface area, which allows more MO dye molecules to attach to the surface and may help to increase the charge-transfer rate [25].

The reusability of the ZnSe nanorod film was also studied. The absorbance spectra of the MO dye solution in the presence of the $\mathrm{ZnSe}$ nanorod film with irradiation for $1.5 \mathrm{~h}$ and $3.0 \mathrm{~h}$ were recorded. After each examination, the $\mathrm{ZnSe}$ nanorod film was immersed into a fresh MO dye solution of the same concentration for another cycle of the photocatalytic experiments. This process was repeated 5 times. The results are given in Figure 6(d) and demonstrate that $\mathrm{ZnSe} \mathrm{nanorod}$ films can be used as a very effective, environmentally safe, and convenient recyclable photocatalyst. A slight decrease in the 


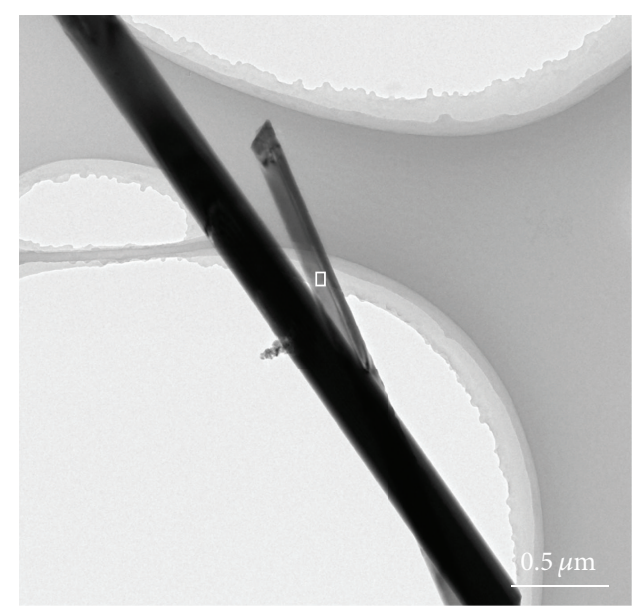

(a)

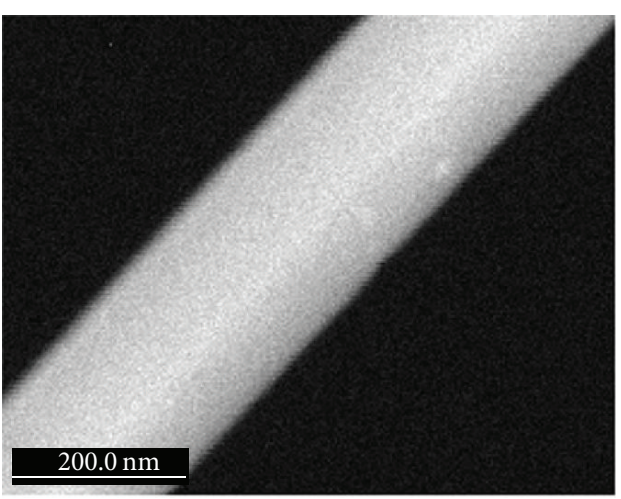

(c)

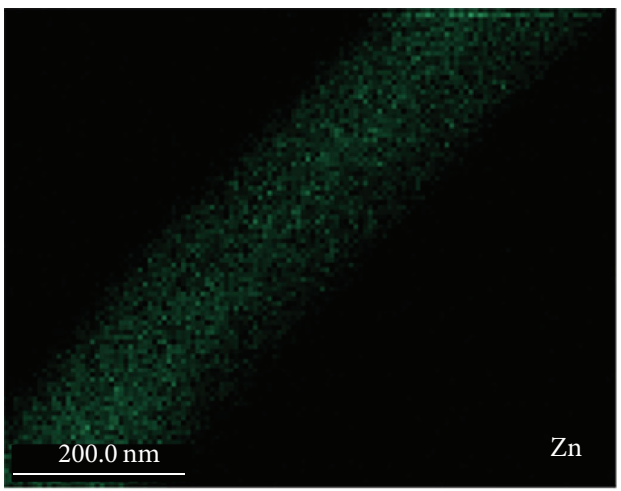

(e)

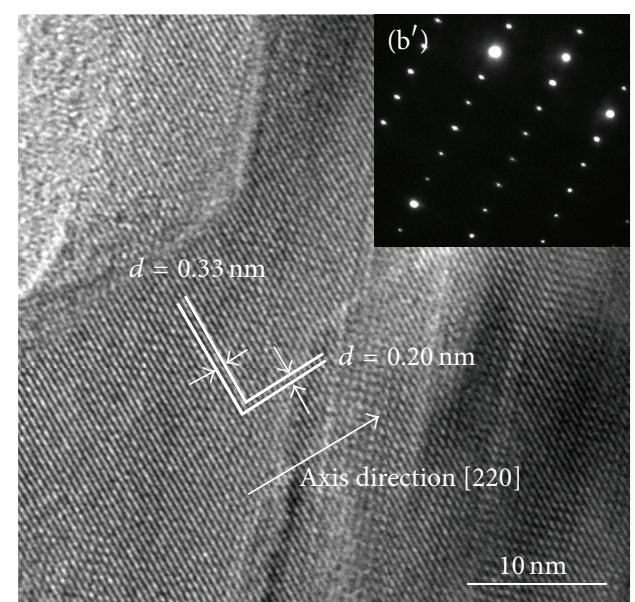

(b)

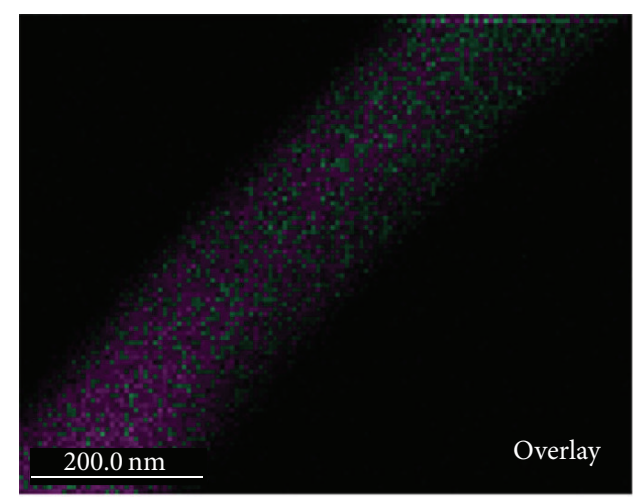

(d)

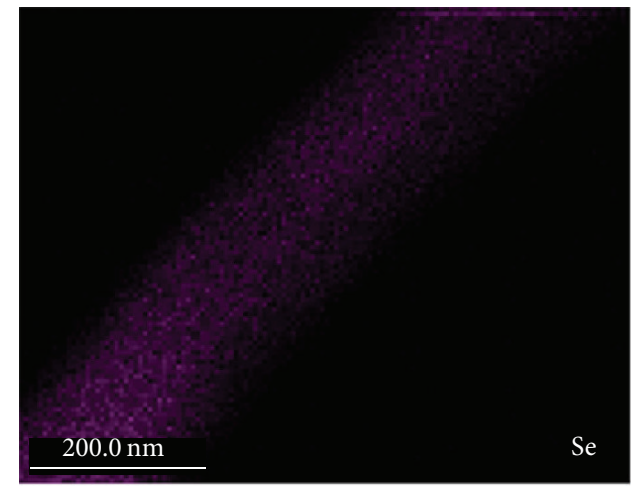

(f)

FIgURE 5: (a) TEM morphology and (b) HRTEM images of ZnSe nanorods taken from the film. The inset in (b) is the corresponding SAED pattern. (c)-(f) are images of the ZnSe nanorods using dark-field SEM and EDS elemental mapping (scale bars, $200 \mathrm{~nm}$ ).

degradation rate was observed and may be attributed to the unavoidable loss of $\mathrm{ZnSe}$ nanorods into solution during the experiment.

\section{Conclusion}

In summary, ZnSe nanorod films were successfully synthesized using a simple two-step synthesis protocol. Dense and randomly oriented $\mathrm{ZnSe}$ nanorods with diameters of $200 \mathrm{~nm}$ and lengths of $15 \mu \mathrm{m}$ were grown on the fused quartz glass substrates. HRTEM, XRD, and EDS patterns confirm the composition and crystalline structure of the $\mathrm{ZnSe}$ nanorod film. These $\mathrm{ZnSe}$ nanorod films were demonstrated as very effective, environmentally safe, and convenient recyclable photocatalysts under ultraviolet light. 

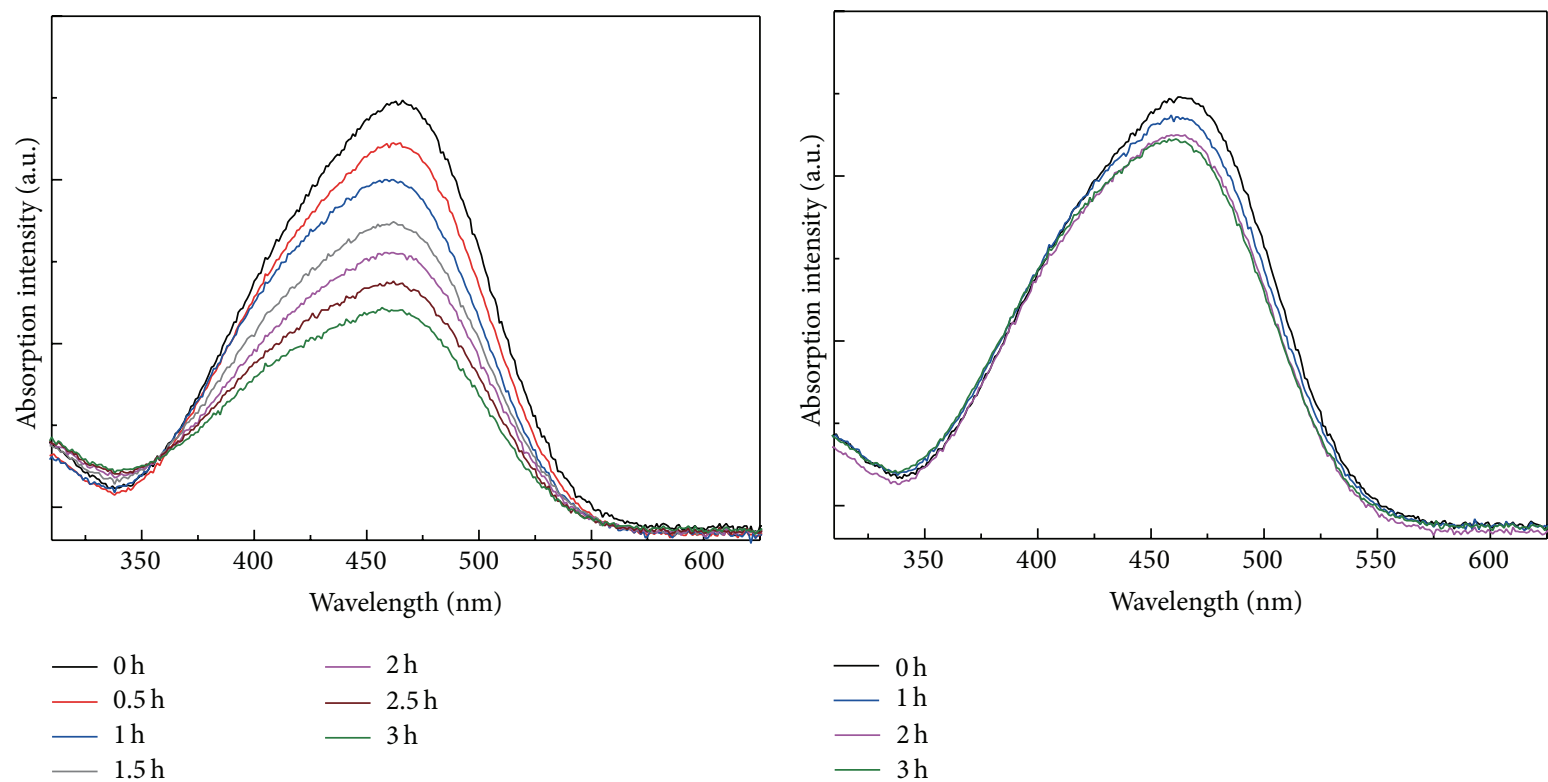

(a)

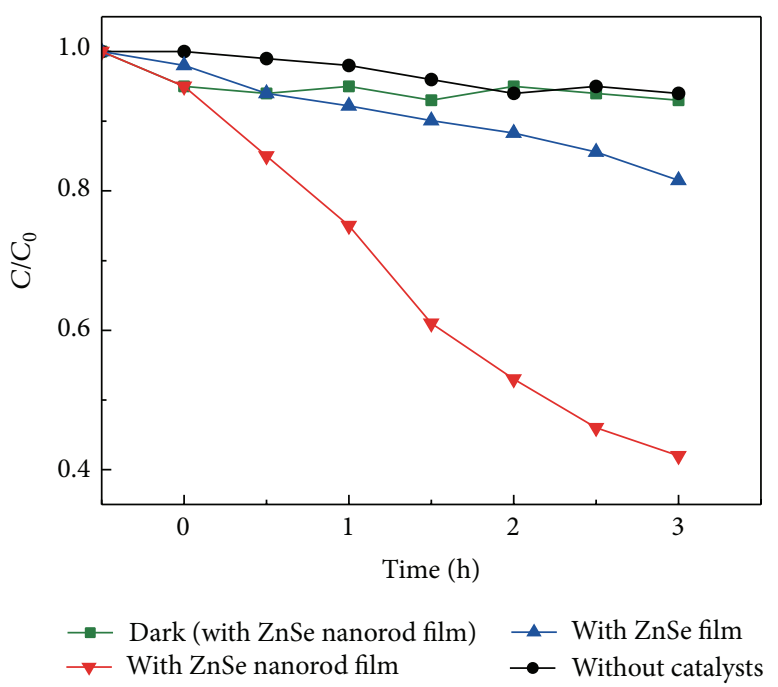

(c)

(b)

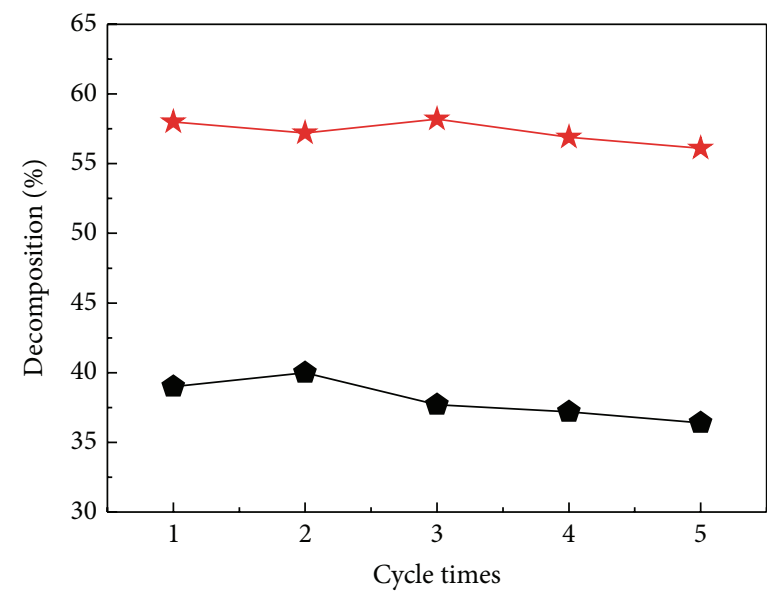

$1.5 \mathrm{~h}$

$3 \mathrm{~h}$

FIGURE 6: Absorption spectrum of the MO dye solution in the presence of the ZnSe nanorod film (12 h) (a) and the ZnSe film (b) under ultraviolet light irradiation for various time durations. (c) Photodegradation curves of the MO dye solution under ultraviolet light in the presence of the ZnSe nanorod film $(12 \mathrm{~h})$, the ZnSe film, and no catalyst and under no ultraviolet light but in the presence of the ZnSe nanorod film (12 h). (d) Degradation change as a function of irradiation time $(1.5 \mathrm{~h}$ and $3.0 \mathrm{~h})$ over 5 cycles. The initial concentration $C_{0}$ was taken to be the same as in (a).

\section{Competing Interests}

The authors declare that there are no competing interests regarding the publication of this paper.

\section{Acknowledgments}

This work was supported by Major Program of National Natural Science Foundation of China (60890200) and National Natural Science Foundation of China (10976017).

\section{References}

[1] A. B. Panda, S. Acharya, and S. Efrima, "Ultranarrow ZnSe nanorods and nanowires: structure, spectroscopy, and one-dimensional properties," Advanced Materials, vol. 17, no. 20, pp. 2471-2474, 2005.

[2] X. Fang, S. Xiong, T. Zhai et al., "High-performance blue/ultraviolet-light-sensitive ZnSe-nanobelt photodetectors," Advanced Materials, vol. 21, no. 48, pp. 5016-5021, 2009.

[3] G. Feng, C. Yang, and S. Zhou, "Nanocrystalline $\mathrm{Cr}^{2+}$-doped ZnSe nanowires laser," Nano Letters, vol. 13, no. 1, pp. 272-275, 2013. 
[4] H. Z. Fu, H. Y. Li, W. Q. Jie, and L. Yang, "The growth and characterization of $\mathrm{ZnSe}$ nanoneedles by a simple chemical vapor deposition method," Journal of Crystal Growth, vol. 289, no. 2, pp. 440-444, 2006.

[5] B. Xiang, H. Z. Zhang, G. H. Li et al., "Green-light-emitting ZnSe nanowires fabricated via vapor phase growth," Applied Physics Letters, vol. 82, no. 19, pp. 3330-3332, 2003.

[6] Y. Chen, L. Wei, G. Zhang, and J. Jiao, "Open structure $\mathrm{ZnO} / \mathrm{CdSe}$ core/shell nanoneedle arrays for solar cells," Nanoscale Research Letters, vol. 7, article 516, 2012.

[7] B. Feng, J. Yang, J. Cao et al., "Growth mechanism, optical and photocatalytic properties of the ZnSe nanosheets constructed by the nanoparticles," Journal of Alloys and Compounds, vol. 555, pp. 241-245, 2013.

[8] J. Yin, G. Feng, and S. Zhou, "Fabrication and photocatalytic property of ZnSe nanosheets," Optoelectronics and Advanced Materials-Rapid Communications, vol. 9, no. 5-6, pp. 682-687, 2015.

[9] F. Cao, W. Shi, L. Zhao et al., "Hydrothermal synthesis and high photocatalytic activity of 3D Wurtzite ZnSe Hierarchical Nanostructures," The Journal of Physical Chemistry C, vol. 112, no. 44, pp. 17095-17101, 2008.

[10] L. Zhang, H. Yang, X. Xie, F. Zhang, and L. Li, "Preparation and photocatalytic activity of hollow ZnSe microspheres via Ostwald ripening," Journal of Alloys and Compounds, vol. 473, no. 1-2, pp. 65-70, 2009.

[11] J. L. Yang, S. J. An, W. I. Park, G.-C. Yi, and W. Choi, "Photocatalysis using $\mathrm{ZnO}$ thin films and nanoneedles grown by metalorganic chemical vapor deposition," Advanced Materials, vol. 16, no. 18, pp. 1661-1664, 2004.

[12] X. Lin, F. Rong, X. Ji, and D. Fu, "Carbon-doped mesoporous $\mathrm{TiO}_{2}$ film and its photocatalytic activity," Microporous and Mesoporous Materials, vol. 142, no. 1, pp. 276-281, 2011.

[13] W. Z. Wang, Y. Geng, P. Yan, F. Y. Liu, Y. Xie, and Y. T. Qian, "Synthesis and characterization of MSe $(\mathrm{M}=\mathrm{Zn}, \mathrm{Cd})$ nanorods by a new solvothermal method," Inorganic Chemistry Communications, vol. 2, no. 3, pp. 83-85, 1999.

[14] N. Kouklin, L. Menon, A. Z. Wong et al., "Giant photoresistivity and optically controlled switching in self-assembled nanowires," Applied Physics Letters, vol. 79, no. 26, pp. 4423-4425, 2001.

[15] R. Solanki, J. Huo, J. L. Freeouf, and B. Miner, "Atomic layer deposition of $\mathrm{ZnSe} / \mathrm{CdSe}$ superlattice nanowires," Applied Physics Letters, vol. 81, article 3864, 2002.

[16] T. Yao, Y. Makita, and S. Maekawa, "Molecular beam epitaxy of $\mathrm{ZnSe}_{x} \mathrm{Te}_{1-x}(0 \precsim x \precsim 1)$," Journal of Crystal Growth, vol. 45, pp. 309-312, 1978.

[17] X. T. Zhang, K. M. Ip, Z. Liu, Y. P. Leung, Q. Li, and S. K. Hark, "Structure and photoluminescence of $\mathrm{ZnSe}$ nanoribbons grown by metal organic chemical vapor deposition," Applied Physics Letters, vol. 84, no. 14, pp. 2641-2643, 2004.

[18] X. T. Zhang, Z. Liu, Y. P. Leung, Q. Li, and S. K. Hark, "Growth and luminescence of zinc-blende-structured $\mathrm{ZnSe}$ nanowires by metal-organic chemical vapor deposition," Applied Physics Letters, vol. 83, no. 26, pp. 5533-5535, 2003.

[19] M.-Z. Xue and Z.-W. Fu, "Electrochemical properties of $\mathrm{LiFePO}_{4}$ cathode thin film fabricated by pulsed laser deposition," Acta Physico-Chimica Sinica, vol. 21, no. 7, pp. 707-710, 2005.

[20] H. Zhang and J. F. Banfield, "Aggregation, coarsening, and phase transformation in $\mathrm{ZnS}$ nanoparticles studied by molecular dynamics simulations," Nano Letters, vol. 4, no. 4, pp. 713-718, 2004.
[21] J. C. Manifacier, J. Gasiot, and J. P. Fillard, "A simple method for the determination of the optical constants $\mathrm{n}, \mathrm{k}$ and the thickness of a weakly absorbing thin film," Journal of Physics E: Scientific Instruments, vol. 9, no. 11, pp. 1002-1004, 1976.

[22] J. E. Williams, V. V. Fedorov, D. V. Martyshkin, I. S. Moskalev, R. P. Camata, and S. B. Mirov, "Mid-IR laser oscillation in $\mathrm{Cr}^{2+}$ : ZnSe planar waveguide," Optics Express, vol. 18, no. 25, pp. 25999-26006, 2010.

[23] N. Kislov, J. Lahiri, H. Verma, D. Y. Goswami, E. Stefanakos, and M. Batzill, "Photocatalytic degradation of methyl orange over single crystalline $\mathrm{ZnO}$ : orientation dependence of photoactivity and photostability of $\mathrm{ZnO}$," Langmuir, vol. 25, no. 5, pp. 33103315, 2009.

[24] L. Zhang, H. Yang, J. Yu et al., "Controlled synthesis and photocatalytic activity of ZnSe nanostructured assemblies with different morphologies and crystalline phases," The Journal of Physical Chemistry C, vol. 113, no. 14, pp. 5434-5443, 2009.

[25] H. J. Yun, H. Lee, J. B. Joo, W. Kim, and J. Yi, "Influence of aspect ratio of $\mathrm{TiO}_{2}$ nanorods on the photocatalytic decomposition of formic acid," The Journal of Physical Chemistry C, vol. 113, no. 8, pp. 3050-3055, 2009. 

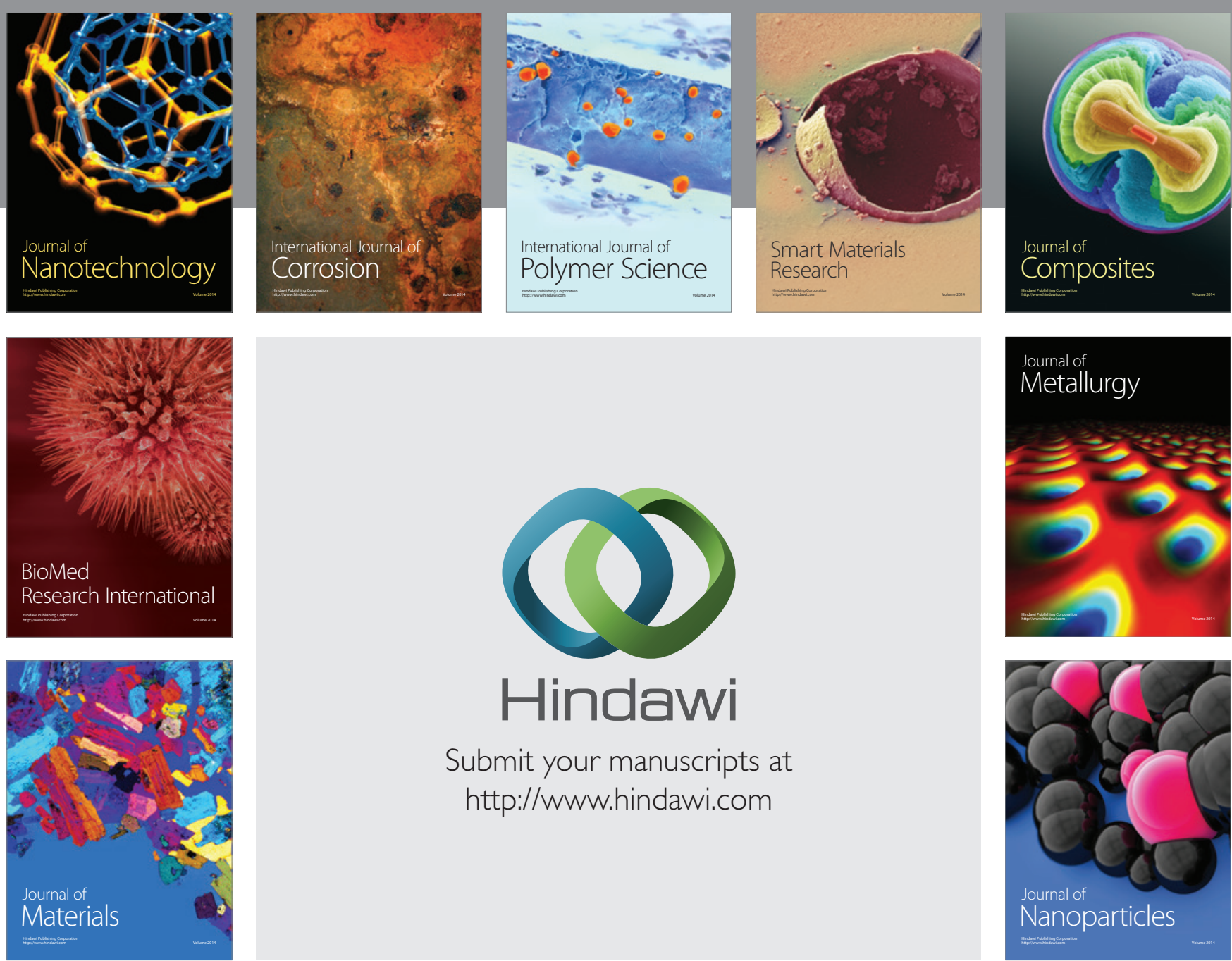

\section{Hindawi}

Submit your manuscripts at

http://www.hindawi.com

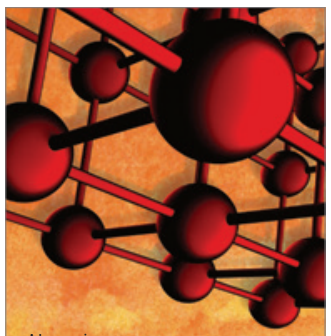

Materials Science and Engineering
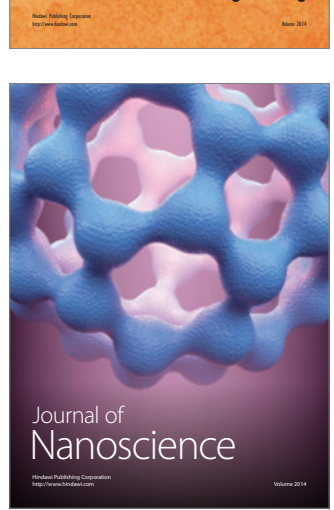
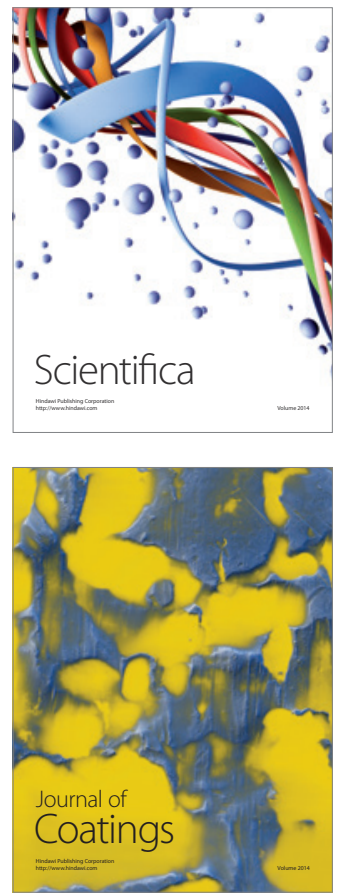
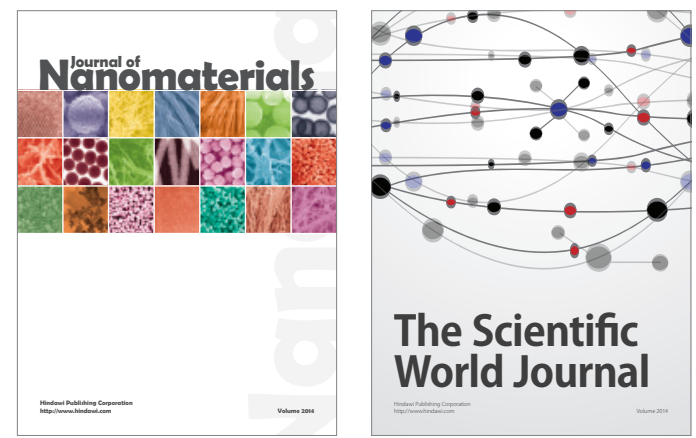

The Scientific World Journal
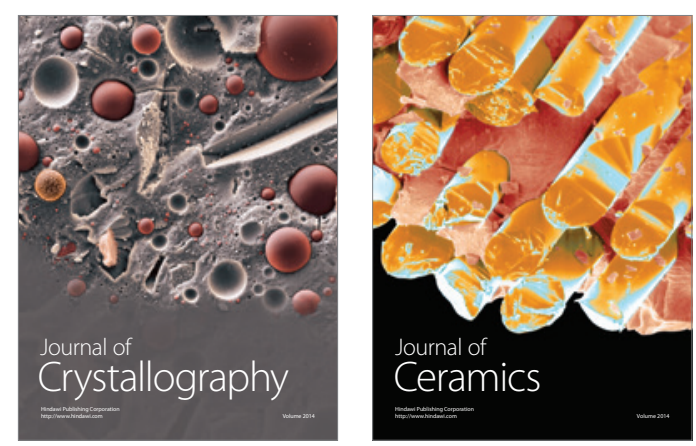
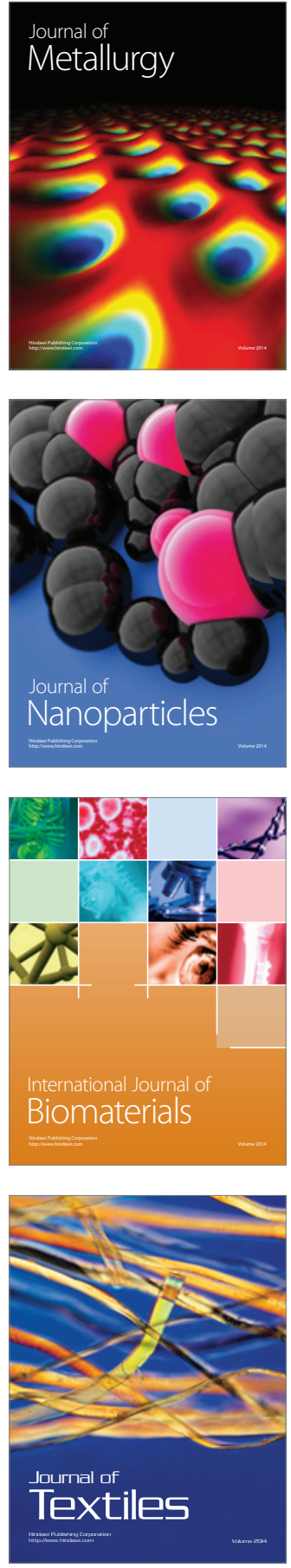